EPSC Abstracts

Vol.14, EPSC2020-666, 2020

Europlanet Science Congress 2020

(C) Author(s) 2020. This work is distributed under

the Creative Commons Attribution 4.0 License.

\title{
Outcome of Collisions in the Early Outer Solar System
}

Jean-Baptiste Vincent ${ }^{1}$, Paula Benavidez ${ }^{2}$, Adriano Campo-Bagatin ${ }^{2}$, Martin Jutzi ${ }^{3}$, Ekkehard Kuehrt $^{1}$, Robert Luther ${ }^{4}$, Patrick Michel ${ }^{5}$, Detlef de Niem ${ }^{1}$, Nilda Oklay ${ }^{6}$, Luca Penasa ${ }^{7}$, and Kai Wünnemann ${ }^{4}$

${ }^{1}$ DLR Institute for Planetary Research, Berlin, Germany (jean-baptiste.vincent@dlr.de)

${ }^{2}$ University of Alicante, Spain

${ }^{3}$ University of Bern, Switzerland

${ }^{4}$ Museum für Naturkunde, Leibniz-Institut für Evolutions- und Biodiversitätsforschung , Berlin, Germany

${ }^{5}$ Laboratoire Lagrange, OCA Nice, France

${ }^{6}$ Independent researcher

${ }^{7}$ Diapartimento di Geoscienze, University of Padova, Italy

\begin{abstract}
The processes leading to the formation of planetary systems leave behind a significant mass of small bodies - up to 35 Earth masses depending on the model [1] - orbiting at large heliocentric distance, and observed around $20 \%$ of Sun-like stars [2]. It is established that those bodies play an important role in the migration of gas giants away from their stars and may be necessary for life to develop on the smaller planets. Yet, the conditions within these primitive populations are not well understood, especially their collisional environment.
\end{abstract}

In the last decade, space missions have brought fascinating new data which challenge our concepts of impacts in the Early Solar System. The mission Rosetta at comet 67P, for instance, has revealed a complex cometary world where collisions, from small to catastrophic, played a significant role. Recent work $[3,4]$ suggests that the topography of cometary nuclei and potential layering are shaped by processes which are primarily ancient. Current erosion does not appear sufficient to create features such as deep pits and tall cliffs over the lifetime of a comet in the Inner Solar System. Could they be due to impacts ? Or early activity in the Centaur phase ?

On a larger scale, dynamical simulations argue that objects like the Jupiter Family Comets may have been totally disrupted by catastrophic collisions. While models show that the high porosity and volatile content of cometary nuclei would survive such impacts, it is not clear whether the deeper structural features like layers can be preserved. From the same data set, different authors come to different conclusions with respect to collisions in the early outer planetary system (summarized in [5]). Furthermore, different modeling approaches $[6,7,8]$ lead to distinct results.

Overall, there is quite some confusion as to how to interpret the cratering record on very porous, icy objects, in the Outer Solar System. This is further complicated by the fact that we may not be able to properly recognize impact features on such bodies.

Here we report on the results obtained by the OCEOSS (Outcome of Collisions in the Early Outer Solar System) ISSI group. The concept of this working group is to bring together experts on 
collisions and cometary morphology. Over the last few years, modelers have developed new numerical simulations to account for cometary-like material. In parallel, thanks to the Rosetta and New Horizon missions, small bodies morphologists have a much better understanding of the type of landform that can exist on comets, and new measurements of the material physical properties.

Our group investigates how morphological features observed today on comets and KBOs could relate to the early collisional environment, beyond the typical crater counting studies.

We will summarize our results along three main topics: Large scale collisions, small scale collisions, and populations.

We find that the outcome of merger collisions, such as the one which may have created comet 67P or KBO Arrokoth can be very different depending on the internal structure of the bodies. If layers are already present before the collision, the impact may lead to shedding and creation of cliffs/terraces resembling what we see on comets.

More energetic impacts would lead to catastrophic disruption, although the fragments may come back together by gravitational interaction, leading to cometary rubble piles. Recent work on this process for asteroids shows that this could lead to the high porosity and potential density variation we observe across bilobate-comets.

On a local scale, we simulated a $\mathrm{km}$ size projectile impacting a $30 \mathrm{~km} \mathrm{KBO}$, which is the expected order of magnitude for creating features such as the largest crater observed on Arrokoth [9]. We find a large diversity of depth-to-diameter ratio (d/D) for craters, depending on the impact and material parameters. We caution against the simple asteroid-like approximation $d / D=10$ for all cases [10], as it can lead to erroneous interpretation of the projectile population when extrapolating from crater shapes only.

We will discuss what our models mean for the interpretation of current spacecraft data, and how this work can support future missions to comets/KBOs, like ESA's Comet Interceptor [11].

\section{References:}

[1] Tsiganis et al., Origin of the orbital architecture of the giant planets of the Solar System, Nature 2005

[2] Wyatt, M., Extrasolar Kuiper belts, in book The Trans-Neptunian Solar System, 2019

[3] Vincent et al, Constraints on cometary surface evolution derived from a statistical analysis of 67P's topography, MNRAS 2017

[4] Birch et al, Geomorphology of comet 67P/Churyumov-Gerasimenko, MNRAS 2017

[5] Weissmann et al, Origin and Evolution of Cometary Nuclei, SSR 2020

[6] de Niem et al, Low velocity collisions of porous planetesimals in the early solar system. Icarus 2018

[7] Jutzi et al, Formation of bi-lobed shapes by sub-catastrophic collisions. A late origin of comet 67P's structure, A\&A 2017

[8] Schwarz et al, Catastrophic disruptions as the origin of bilobate comets, Nature Astronomy 2018

[9] Spencer et al, The geology and geophysics of Kuiper Belt object (486958) Arrokoth, Science 2020

[10] Bottke et al, Interpreting the Cratering History of Bennu, Ryugu, and Other SpacecraftExplored Asteroids, EPSC 2019

[11] Snodgrass, C. \& Jones, G., The European Space Agency's Comet Interceptor lies in wait, Nature Communications 2019 


\section{Acknowledgements}

The OCEOSS team thanks the International Space Science Institute (ISSI Bern), which made this collaboration possible. 